\title{
Prevalencia de factores de riesgo para diabetes mellitus, según escala Findrisc julio a diciembre de 2018
}

Ledo. Francisco J. Coronado D.

\section{Resumen}

La diabetes mellitus tipo 2 (DM2) es uno de los mayores problemas de salud latinoamericana, donde, de 371 millones de adultos en la región, 26 millones (7\%) padecen DM2. Es más alta la incidencia de la misma en países de bajos ingresos. En cuanto mayor sea el lapso entre el inicio de la enfermedad con el diagnóstico y tratamiento de la DM en los pacientes, peores son las repercusiones para la salud. La finalidad de las encuestas de tamizaje es evaluar el riesgo de padecimiento de diabetes en pacientes, sin someterlo a pruebas de laboratorio, que es un método sensible, económico y replicable en el primer nivel de salud, para el diagnóstico oportuno de la enfermedad (1), (2). Los objetivos fueron identificar la prevalencia de factores de riesgo para diabetes mellitus, a través de la escala Findrisc en la población de la comunidad Buena Vista II, San Juan Ostuncalco. El diseño consistió en un estudio descriptivo, observacional, transversal. Se realizó en comunidad Buena Vista II, San Juan Ostuncalco, Quetzaltenango. Los materiales y métodos utilizados consistieron en un estudio realizado a través de 239 encuestas a personas mayores de 18 años, residentes en aldea Buena Vista II, San Juan Ostuncalco, Quetzaltenango, durante los meses de julio a diciembre del 2018. Como resultado se encontró que los factores de riesgo de la escala Findrisc para la población total de mayor a menor prevalencia fueron: «no consumo diario de frutas y verduras» (86 \%), «no realizar al menos 150 minutos de actividad física moderada a la semana» (83\%), «perímetro de cintura >94 (hombres) >80 (mujeres)» (82\%), «IMC 25 (sobrepeso y obesidad)» (67\%), «antecedente de diagnóstico de diabetes (tipo 1 ○ 2) a algún familiar o pariente próximo» (28\%), «edad $\geq$ 45 años» (19\%), «si ha tomado o toma regularmente medicamentos para la presión (hipertensión)» (14\%), «si le han encontrado la glucosa sanguínea alta» (6\%). El 0 \% de la población con alto riesgo en la escala Findrisc, presentó una glicemia capilar en ayunas normal (100 mg/dl). Lo que lleva a las siguientes conclusiones que los factores de riesgo para el desarrollo de diabetes mellitus utilizados en la escala Findrisc, fueron predominantemente factores de riesgo modificables, como: obesidad abdominal, el sedentarismo, el IMC $>25 \mathrm{~kg} / \mathrm{m}^{2}$ y el mal patrón alimenticio.

Palabras clave: diabetes mellitus, factores de riesgo, Findrisc. 


\section{Prevalence of risk factors for diabetes mellitus, calculated with FINDRISC Prediction Tool} Fuly to December, 2018

\section{Abstract}

Diabetes mellitus type 2 (DM2) poses one of the most serious health problems in Latin America, where 26 million (7 \%) out of 371 million adults have DM2, with a higher prevalence in lower income countries. The greater the time lapse is from the onset of the disease to its diagnosis and treatment, the worse will health repercussions be. The objective of a screening survey is to evaluate the risk of diabetes in patients without having to do lab tests. This method is sensible, economic and replicable at the first level of health and provides a timely diagnosis of the disease. For the present study, the FINDRISC Diabetes Risk Calculator was used in order to identify the prevalence of risk factors in the community of Buena Vista II, San Juan Ostuncalco, Quetzaltenango. The design of the study was descriptive, observational and cross-sectional, based on a survey consisting of 239 interviews to residents of Buena Vista II, age 18 or more, performed from July through December 2018. The Findrisk-based survey established that prevalence of risk factors for the total population was the following: «no fruit and vegetable consumption on a daily basis» (86\%); "no moderate physical activity at least 150 minutes weekly» (83\%); «waist perimeter >94 (males) >80 (females)» (82\%); «BMI 25 (overweight and obesity)» (67\%); «history of diabetes (type 1 or 2) in a relative or close family member» (28\%); "age $\geq 45$ years» (19 $\%)$; "takes or has regularly taken hypertension medicine » (14\%); «has been diagnosed with a high glucose level in blood " (6\%). Among the high risk population calculated with Findrisk, none of the cases $(0 \%)$ presented normal capilar glycemia $(100 \mathrm{mg} / \mathrm{dl})$ before food intake. These results led to the conclusion that based on the Findrisk scale, the risk factors for developing diabetes mellitus consist mostly of modifiable factors such as abdominal obesity, a sedentary life, a BMI $>25 \mathrm{~kg} / \mathrm{m}^{2}$ and a poor diet.

Key words: diabetes mellitus, risk factors, Findrisc.

\section{Introducción}

La Diabetes Mellitus (DM) es una enfermedad que se caracteriza por presentar hiperglicemias, como consecuencia de una alteración hormonal en la secreción o acción de la insulina (6). La DM se puede caracterizar en diversos tipos etiológicos, sin embargo, el aumento de casos en las últimas décadas se ve que son directamente proporcionales al incremento de patrones de estilo de vida no saludables (5). Findrisc es un cuestionario que consta de 8 preguntas, cada una con una puntuación, que predice la probabilidad de desarrollar DM2 en los próximos 10 años (3). Esta evalúa: edad, índice de masa corporal (IMC), circunferencia abdominal, antecedentes médicos y familiares de hipertensión o hiperglicemias, actividad física y consumo de frutas, verduras y hortalizas. Con la ventaja que puede ser auto administrado y que permite detectar personas de alto riesgo en la población general y en la práctica clínica habitual (2).

\section{Antecedentes}

Las guías internacionales concuerdan que el estándar de oro para el diagnóstico de DM, es a través de pruebas de glucemia sérica, que coinciden 
con que estas pruebas deben ser realizadas como tamizaje en pacientes mayores de 45 años o que cumplen factores de riesgos asociados a DM (1), (2).

Se estima que el $50 \%$ de la población general con diabetes mellitus desconoce su diagnóstico, por lo que la importancia del tamizaje es clara (1), (2), (4). Las encuestas de tamizaje son desarrolladas y adaptadas para diversas poblaciones, con el propósito de evaluar el riesgo de padecer diabetes en pacientes sin someterlo a pruebas de laboratorio «curva de tolerancia a la glucosa oral (TTGO), hemoglobina glicosilada (HbA1c) o glucosa sérica en ayunas (Gb)» a excepción de la población catalogada de alto riesgo (1), (3), (4).

\section{Instrumento}

Los datos de este estudio se obtuvieron a través de la entrevista personal a los participantes, se seleccionaron todas las viviendas y dentro de cada una se escogió aleatoriamente a una persona mayor de 18 años. Datos sociodemográficos preguntas directas sobre edad, género, etnicidad, escolaridad, vivienda y servicios básicos. Datos de estilo de vida: se obtuvo a través de la entrevista personal, que incluyó caracterización de la actividad física, del consumo de frutas, verduras y hortalizas, antecedentes médicos de hiperglicemia e hipertensión arterial y presencia de antecedente familiar de DM. Así como el registro de los resultados de la evaluación antropométrica y de glicemia capilar obtenida.

\section{Recolección de datos}

El trabajo de campo se llevó acabo de julio a diciembre del 2018, en la comunidad de Buena Vista II, San Juan Ostuncalco, Quetzaltenango. Se seleccionó el municipio y comunidad de trabajo, por conveniencia, según las opciones brindadas por el programa de medicina comunitaria, de la Universidad Rafael Landívar. Se reajustó el tamaño de muestra utilizando de fuente el Censo de Buena Vista II, realizado durante 2015/2016.

Se seleccionó la muestra, tomando en cuenta: variabilidad desconocida, nivel de confianza de $95 \%$, error de $5 \%$, y una población adulta total 239 personas. Previo al inicio del estudio se informó a los participantes, los beneficios y riesgos en su participación voluntaria, se autorizó la participación del estudio por medio del consentimiento informado. Se llevó a cabo la entrevista dentro de cada vivienda escogida y se llenaron las boletas de recolección de datos. Se citó a toda persona entrevistada con puntuación mayor de 15 puntos en la escala Findrisc, para toma de glicemia capilar en ayunas, de no ser así, se procedía a la búsqueda de la persona en su vivienda. A todo(a) participante que presentó una glicemia capilar en ayunas mayor a $100 \mathrm{mg} / \mathrm{dl}$ se le refirió a un segundo y/o tercer nivel de salud, para confirmar diagnóstico de DM, en conjunto a la creación de un expediente médico de seguimiento en la unidad mínima de salud de la localidad. 


\section{Análisis estadístico}

Los datos recolectados de las boletas, fueron trasladados a una hoja electrónica en archivo Excel. Se ordenaron todas las boletas en formato electrónico y se procedió a realizar el análisis e interpretación de datos, por distribución de frecuencia porcentajes y niveles de riesgo. Se analizaron los datos de todas las boletas registradas en formato electrónico. El análisis fue dirigido en cumplimiento de los objetivos de la investigación. Se elaboraron cuadros y gráficas para analizar y presentar los datos obtenidos de una manera clara.

\section{Resultados}

Tabla 1

Características sociodemográficas

\begin{tabular}{|c|c|c|c|c|}
\hline \multicolumn{2}{|c|}{$\begin{array}{c}\text { Características } \\
\text { Sociodemográficas } \\
\qquad n=239\end{array}$} & $\begin{array}{c}f \\
n=239\end{array}$ & $\%$ & $\begin{array}{c}\text { IC } \\
(95 \%)\end{array}$ \\
\hline \multirow{2}{*}{ Género } & Femenino & 209 & 87.4 & $(83,92)$ \\
\hline & Masculino & 30 & 12.6 & $(8.17)$ \\
\hline \multirow{2}{*}{ Etnicidad } & Ladino & 34 & 14.2 & $(10,19)$ \\
\hline & Maya & 205 & 85.8 & $(81,90)$ \\
\hline \multirow{5}{*}{ Escolaridad } & No estudió & 48 & 20.1 & $(15,25)$ \\
\hline & Primaria & 101 & 42.3 & $(36,49)$ \\
\hline & Básicos & 40 & 16.7 & $(12,21)$ \\
\hline & Diversificado & 41 & 17.2 & $(12,22)$ \\
\hline & Universidad & 9 & 3.8 & $(1,6)$ \\
\hline \multirow{3}{*}{$\begin{array}{l}\text { Servicios } \\
\text { básicos }\end{array}$} & Luz eléctrica & 229 & 95.8 & $(93,98)$ \\
\hline & Agua potable & 232 & 97.1 & $(95,99)$ \\
\hline & Letrina & 221 & 92.5 & $(89,96)$ \\
\hline \multirow{5}{*}{$\begin{array}{l}\text { Material } \\
\text { de piso }\end{array}$} & Azulejo & 2 & 0.8 & $(0.3,2)$ \\
\hline & Cerámico & 69 & 28.9 & $(23,35)$ \\
\hline & Granito & 3 & 1.3 & $(0,3)$ \\
\hline & Tierra & 22 & 9.2 & $(6,13)$ \\
\hline & Torta de cemento & 143 & 59.8 & $(54,66)$ \\
\hline \multirow{2}{*}{$\begin{array}{l}\text { Material de } \\
\text { pared }\end{array}$} & Block & 237 & 99.2 & $(98,100)$ \\
\hline & Habita & 2 & 0.8 & $(0,2)$ \\
\hline \multirow{2}{*}{$\begin{array}{l}\text { Material de } \\
\text { techo }\end{array}$} & Lámina & 140 & 58.6 & $(52,65)$ \\
\hline & Terraza & 99 & 41.4 & $(35,48)$ \\
\hline
\end{tabular}

Fuente: informe final de tesis. 
Tabla 2

Riesgo por edad, según escala Findrisc

\begin{tabular}{llll}
\multicolumn{1}{c}{ Distribución de edad } & $f$ & $\%$ & $\mathrm{IC}$ \\
18 a 24 años & $\mathrm{n}=239$ & & $(95 \%)$ \\
25 a 29 años & 74 & 31 & $(25,37)$ \\
30 a 34 años & 32 & 13 & $(9,18)$ \\
35 a 39 años & 31 & 13 & $(9,17)$ \\
40 a 44 años & 33 & 14 & $(9,18)$ \\
45 a 49 años & 23 & 10 & $(6,13)$ \\
50 a 54 años & 13 & 5 & $(3,8)$ \\
55 a 59 años & 9 & 4 & $(1,6)$ \\
60 a 64 años & 7 & 3 & $(0,3)$ \\
65 a 70 años & 2 & 1 & $(0,1)$ \\
$>70$ años & 0 & 0 & $(0)$
\end{tabular}

Fuente: informe final de tesis.

Figura 1. Distribución de estado nutricional

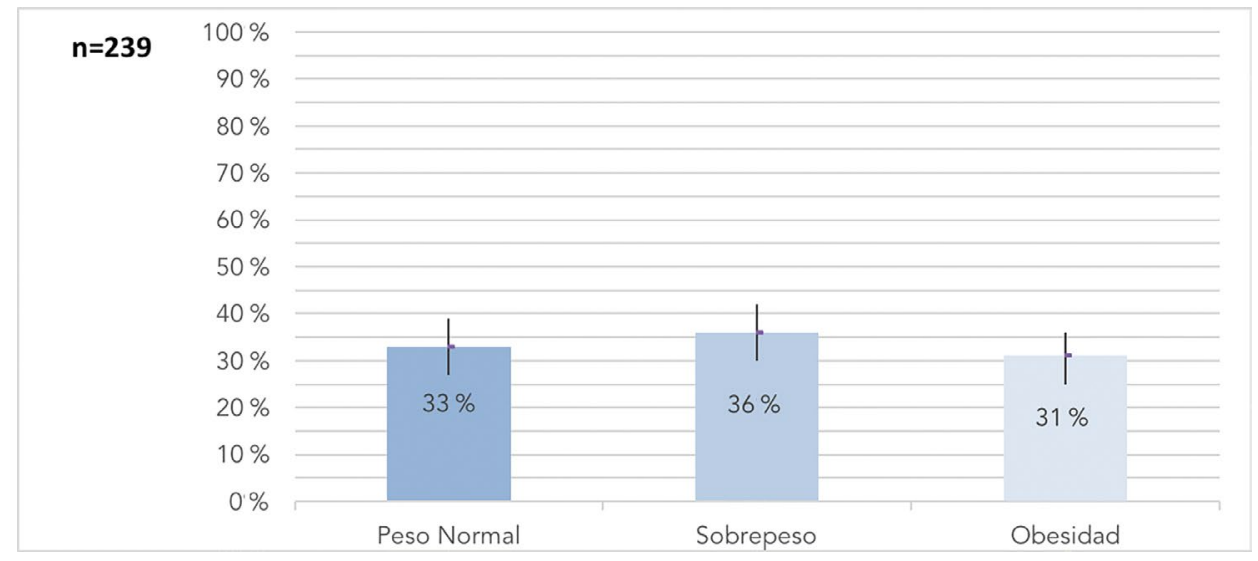

Fuente: informe final de tesis. 
Tabla 3

Distribución de estilos de vida de la población estudiada en relación con el estado nutricional

\begin{tabular}{|c|c|c|c|c|c|c|c|c|c|c|c|c|}
\hline \multirow{2}{*}{$\begin{array}{c}\text { IMC Total } \\
n=239\end{array}$} & \multicolumn{3}{|c|}{$<25 \mathrm{~kg} / \mathrm{m}^{2}$} & \multicolumn{3}{|c|}{$>25 \mathrm{~kg} / \mathrm{m}^{2}$} & \multicolumn{3}{|c|}{25 a $30 \mathrm{~kg} / \mathrm{m}^{2}$} & \multicolumn{3}{|c|}{$>30 \mathrm{~kg} / \mathrm{m}^{2}$} \\
\hline & $f$ & $\%$ & $\begin{array}{c}\text { IC } \\
(95 \%)\end{array}$ & $f$ & $\%$ & $\begin{array}{c}\text { IC } \\
(95 \%)\end{array}$ & $f$ & $\%$ & $\begin{array}{c}\text { IC } \\
(95 \%)\end{array}$ & $f$ & $\%$ & $\begin{array}{c}\text { IC } \\
(95 \%)\end{array}$ \\
\hline \multicolumn{13}{|l|}{ Actividad física } \\
\hline No realiza & 53 & 22 & $(17,27)$ & 109 & 46 & $(39,52)$ & 52 & 22 & $(17,27)$ & 57 & 24 & $(18,29)$ \\
\hline Si realiza & 27 & 11 & $(7,15)$ & 38 & 16 & $(11,21)$ & 25 & 10 & $(7,14)$ & 13 & 5 & $(3,8)$ \\
\hline $\begin{array}{l}<150 \\
\text { minutos/semanales }\end{array}$ & 14 & 6 & $(3,9)$ & 18 & 8 & $(4,11)$ & 5 & 2 & $(0,4)$ & 2 & 1 & $(0,2)$ \\
\hline $\begin{array}{l}>150 \\
\text { minutos/semanales }\end{array}$ & 13 & 5 & $(3,8)$ & 20 & 8 & $(5,12)$ & 12 & 5 & $(2,8)$ & 8 & 3 & $(1,6)$ \\
\hline \multicolumn{13}{|l|}{$\begin{array}{l}\text { Consumo de frutas, } \\
\text { verduras y hortalizas }\end{array}$} \\
\hline Diario & 12 & 5 & $(2,8)$ & 28 & 12 & $(8,16)$ & 18 & 8 & $(4,11)$ & 10 & 4 & $(2.7)$ \\
\hline No consume & 68 & 28 & $(23,34)$ & 130 & 54 & $(48,61)$ & 67 & 28 & $(22,34)$ & 63 & 26 & $(21,32)$ \\
\hline \multicolumn{13}{|l|}{ Aguas edulcoradas } \\
\hline Diario & 25 & 10 & $(7,14)$ & 79 & 33 & $(27,39)$ & 38 & 16 & $(11,21)$ & 41 & 17 & $(12,22)$ \\
\hline No consume & 55 & 23 & $(18,28)$ & 79 & 33 & $(27,39)$ & 47 & 20 & $(15,25)$ & 32 & 13 & $(9,18)$ \\
\hline
\end{tabular}

Fuente: informe final de tesis.

Figura 2. Distribución de perímetro abdominal

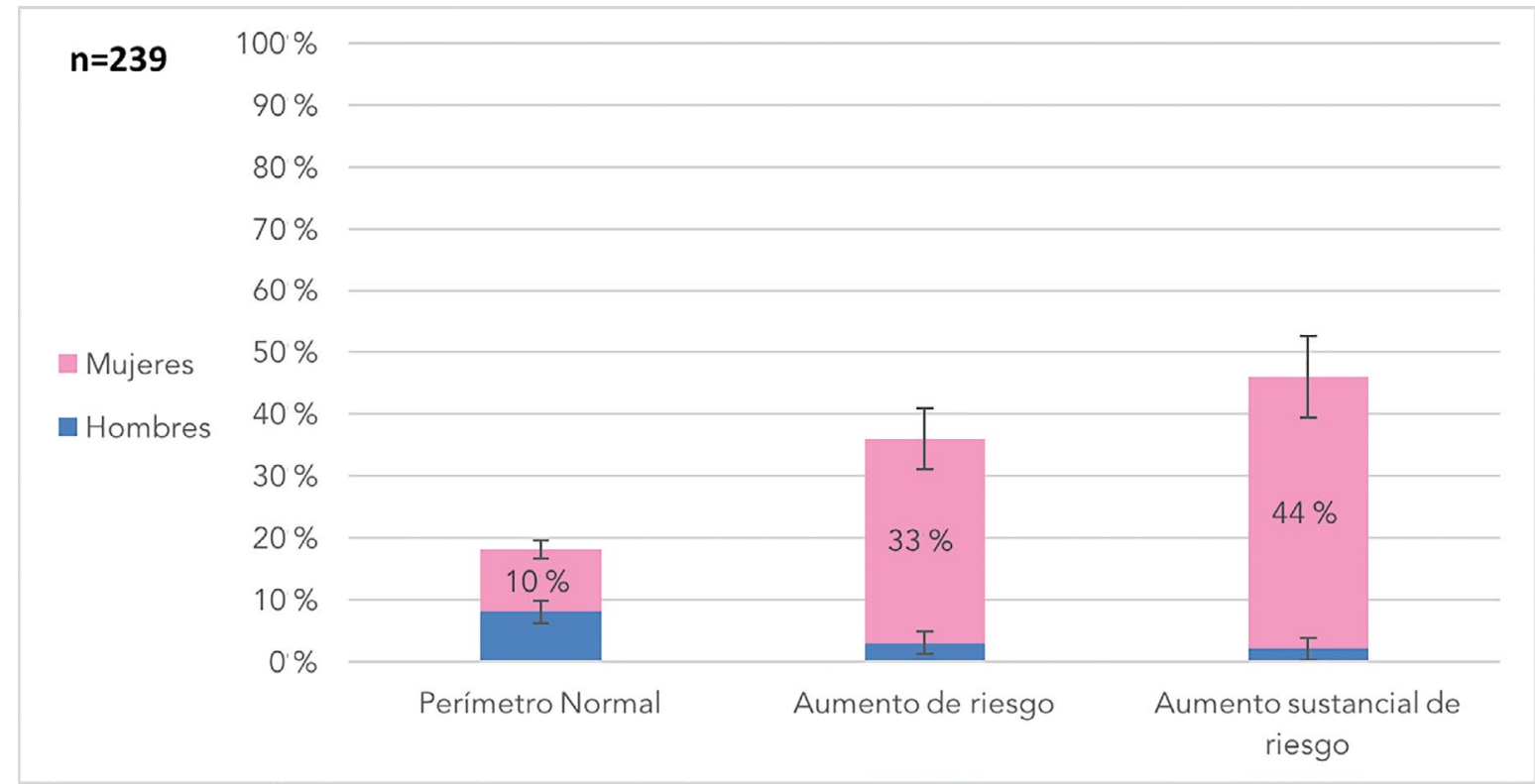

Fuente: informe final de tesis. 
Tabla 4

Distribución de estilos de vida de la población femenina estudiada en relación con al perímetro abdominal

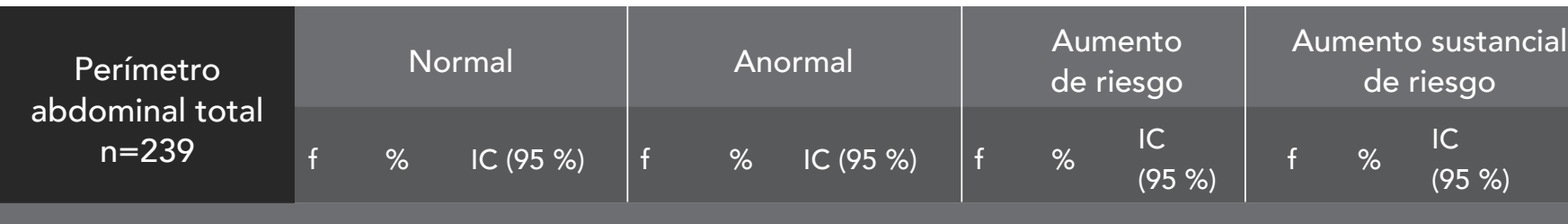

Actividad física

\begin{tabular}{|c|c|c|c|c|c|c|c|c|c|c|c|c|}
\hline No realiza & 19 & 8 & $(5,11)$ & 136 & 57 & $(51,63)$ & 63 & 26 & $(21,32)$ & 73 & 31 & $(25,36)$ \\
\hline Sí realiza & 25 & 10 & $(7,14)$ & 48 & 20 & $(15,25)$ & 26 & 11 & $(7,15)$ & 22 & 9 & $(6,13)$ \\
\hline $\begin{array}{l}<150 \\
\text { minutos/semanales }\end{array}$ & 30 & 13 & $(8,17)$ & 21 & 9 & $(5,12)$ & 9 & 4 & $(1,6)$ & 3 & 1 & $(0,3)$ \\
\hline $\begin{array}{l}>150 \\
\text { minutos/semanales }\end{array}$ & 20 & 8 & $(5,11)$ & 18 & 8 & $(4,11)$ & 8 & 3 & $(1,6)$ & 10 & 4 & $(2,7)$ \\
\hline
\end{tabular}

Consumo de

frutas, verduras

y hortalizas

\begin{tabular}{l|lll|lll|lll|lll} 
Diario & 10 & 4 & $(2,7)$ & 11 & 5 & $(2,7)$ & 0 & 0 & $(0)$ & 11 & 5 & $(2,7)$ \\
No consume & 15 & 6 & $(3,9)$ & 20 & 8 & $(5,11)$ & 10 & 4 & $(2,7)$ & 10 & 4 & $(2,7)$ \\
\hline Aguas edulcoradas & 16 & 7 & $(4,10)$ & 81 & 34 & $(28,40)$ & 32 & 13 & $(9,18)$ & 49 & 21 & $(15,26)$ \\
Diario & 16 & & & & & & & & & & & \\
No consume & 28 & 12 & $(8,16)$ & 103 & 43 & $(37,49)$ & 57 & 24 & $(18,29)$ & 46 & 19 & $(14,24)$
\end{tabular}

Fuente: informe final de tesis.

Figura 3. Distribución de los antecedentes médicos y familiares

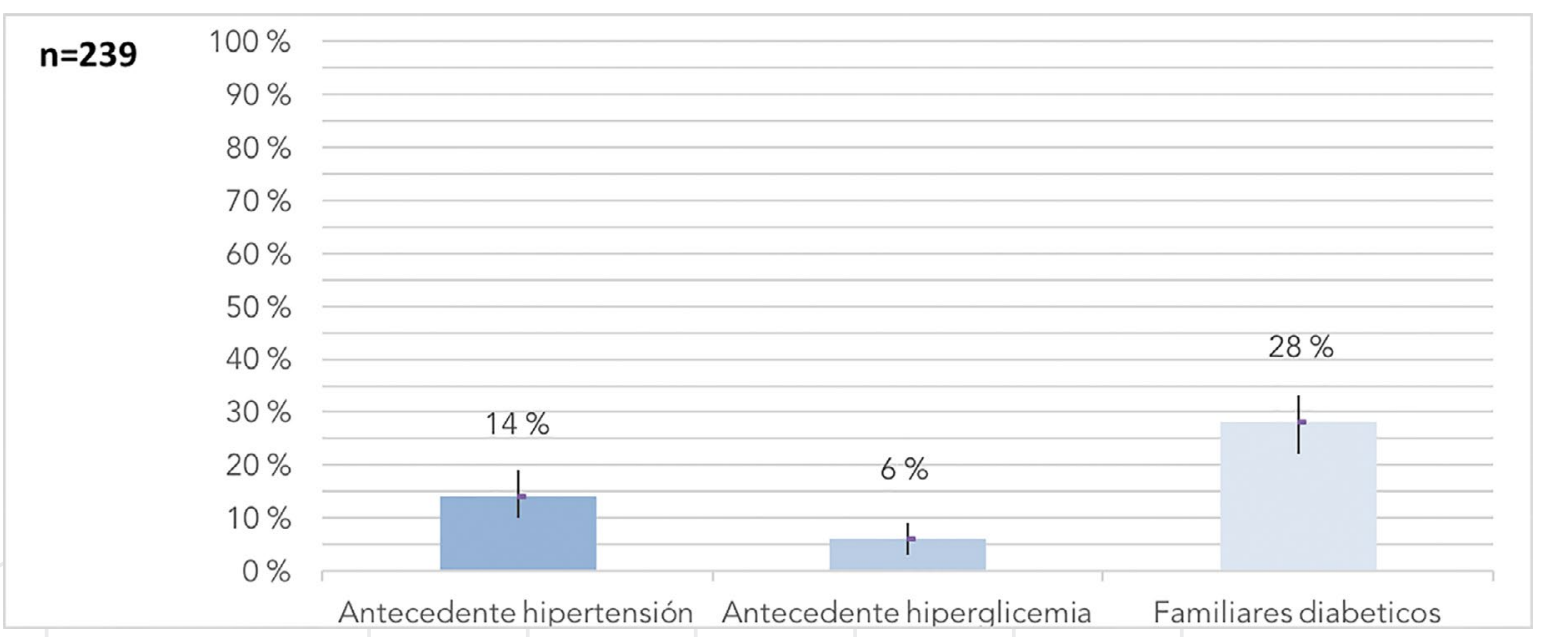

Fuente: informe final de tesis. 
Distribución femenina, según sus características clínicas

\begin{tabular}{|c|c|c|c|c|}
\hline \multicolumn{2}{|c|}{ Características clínicas de la población femenina } & $\begin{array}{l}f \\
n=209\end{array}$ & $\%$ & $\begin{array}{l}\text { IC } \\
(95 \%)\end{array}$ \\
\hline \multirow[t]{4}{*}{ Edad } & $<45$ años & 169 & 81 & $(76,86)$ \\
\hline & Entre 45 a 54 años & 19 & 9 & $(5,13)$ \\
\hline & Entre 55 a 64 años & 8 & 4 & $(1,6)$ \\
\hline & $>65$ años & 13 & 6 & $(3,9)$ \\
\hline \multirow[t]{3}{*}{ IMC } & $<25 \mathrm{Kg} / \mathrm{m}^{2}$ & 69 & 33 & $(27,39)$ \\
\hline & Entre 25 y $30 \mathrm{Kg} / \mathrm{m}^{2}$ & 75 & 36 & $(29,42)$ \\
\hline & $>30 \mathrm{Kg} / \mathrm{m}^{2}$ & 65 & 31 & $(25,37)$ \\
\hline \multirow[t]{3}{*}{ Perímetro abdominal } & Perímetro normal & 25 & 12 & $(8,16)$ \\
\hline & Aumento de riesgo & 94 & 45 & $(38,52)$ \\
\hline & Aumento sustancial de riesgo & 90 & 43 & $(36,50)$ \\
\hline \multirow{2}{*}{$\begin{array}{l}\text { Realizan }>150 \text { minutos/ } \\
\text { semanales de actividad física } \\
\text { moderada }\end{array}$} & No & 182 & 87 & $(83,92$ \\
\hline & Sí & 27 & 13 & $(8,17)$ \\
\hline Consumo de frutas & Diariamente & 50 & 24 & $(18,30)$ \\
\hline Consumo de verduras & Diariamente & 51 & 24 & $(18,30)$ \\
\hline Consumo de hortalizas & Diariamente & 50 & 24 & $(18,30)$ \\
\hline \multirow[t]{3}{*}{ Consumo de aguas edulcoradas } & No consume & 44 & 21 & $(16,27)$ \\
\hline & Ocasionalmente & 78 & 37 & $(31,44)$ \\
\hline & Diariamente & 87 & 42 & $(35,48)$ \\
\hline \multirow[t]{2}{*}{ Antecedentes médicos } & Hipertensión Arterial & 30 & 14 & $(10,19$ \\
\hline & Hiperglicemia & 13 & 6 & $(3,9)$ \\
\hline \multirow[t]{2}{*}{ Familiar diabético } & Sí & 58 & 28 & $(22,34)$ \\
\hline & No & 151 & 63 & $(66,78)$ \\
\hline
\end{tabular}

Fuente: informe final de tesis. 
Figura 4. Prevalencía de factores de riesgo para diabetes mellitus según escala de Findrisc

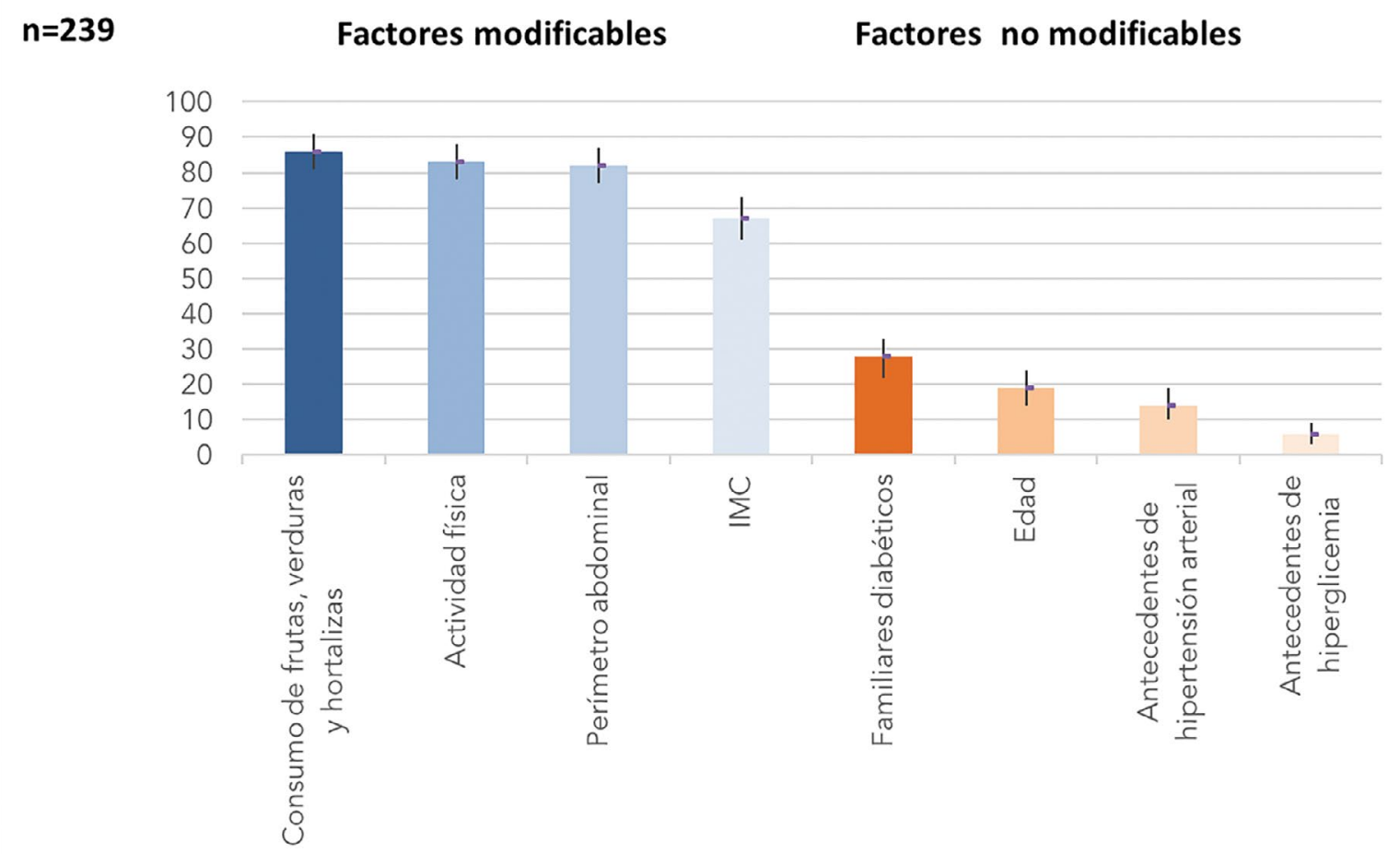

Fuente: informe final de tesis.

Figura 5. Distribución de riesgo según la escala Findrisc

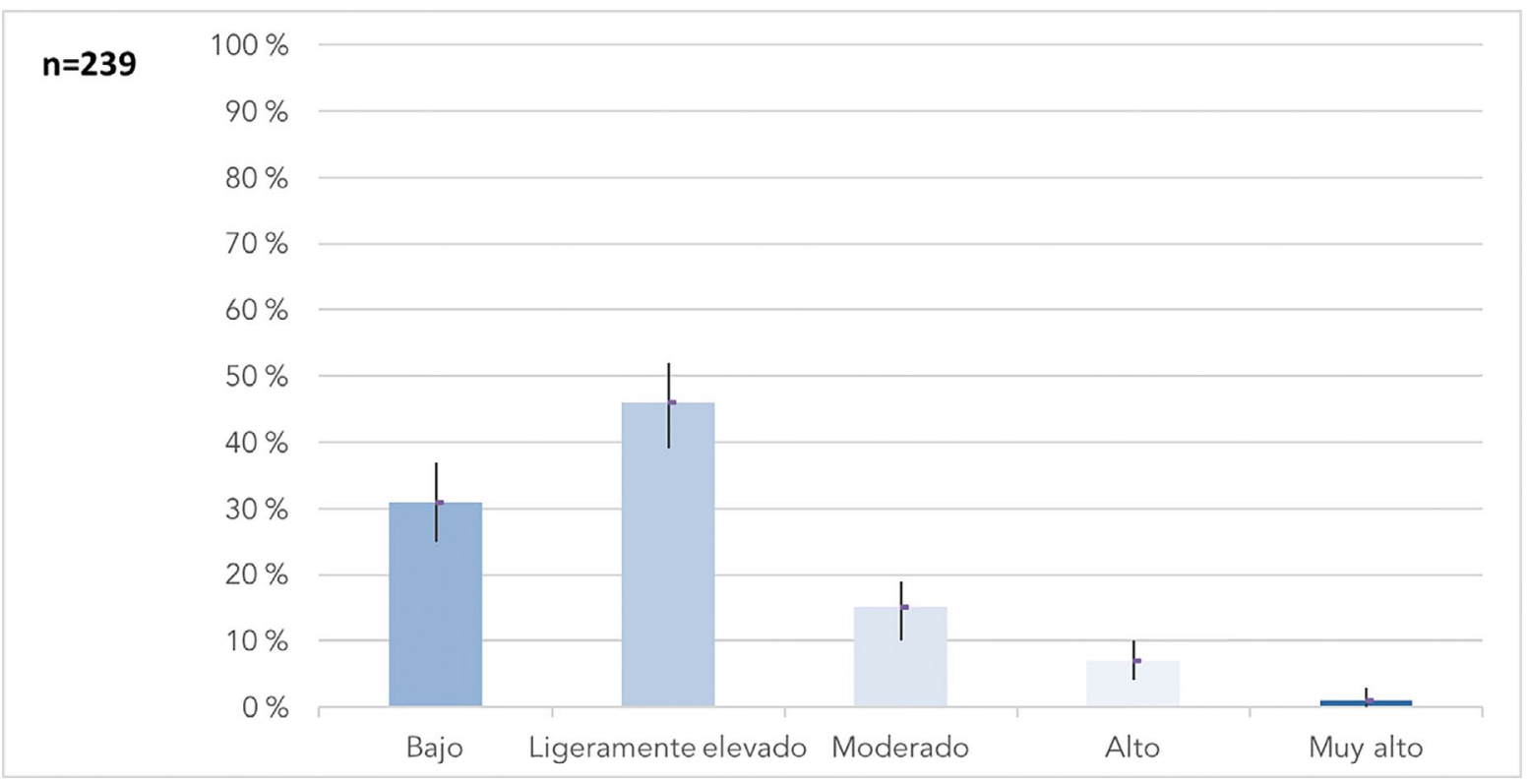

Fuente: informe final de tesis. 
Figura 6. Distribución de consumo de frutas, verduras, hortalizas, y aguas edulcoradas

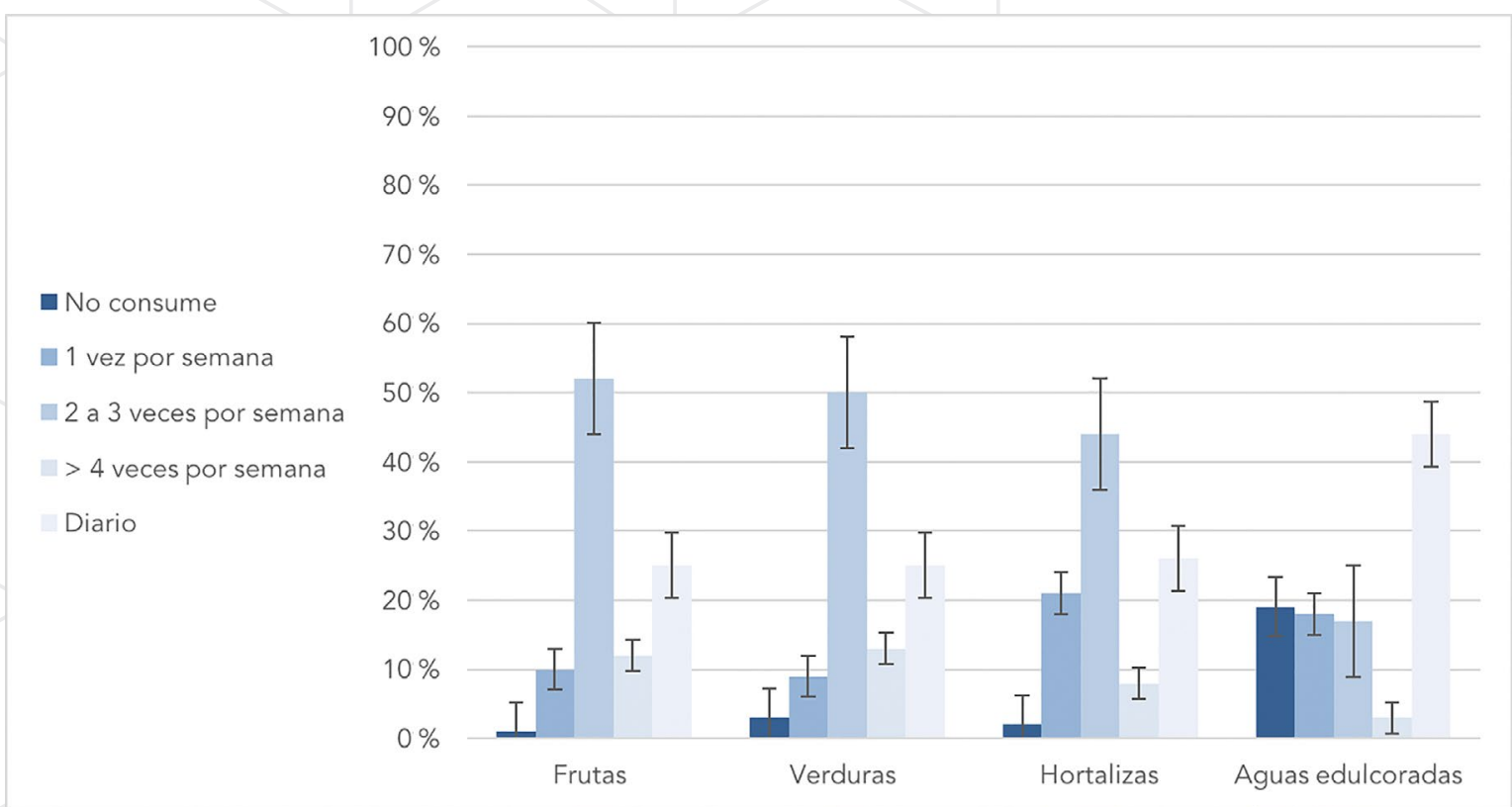

Fuente: informe final de tesis.

Figura 7. Distribución de glicemia en población con puntuación de Findrisc > a 15 puntos

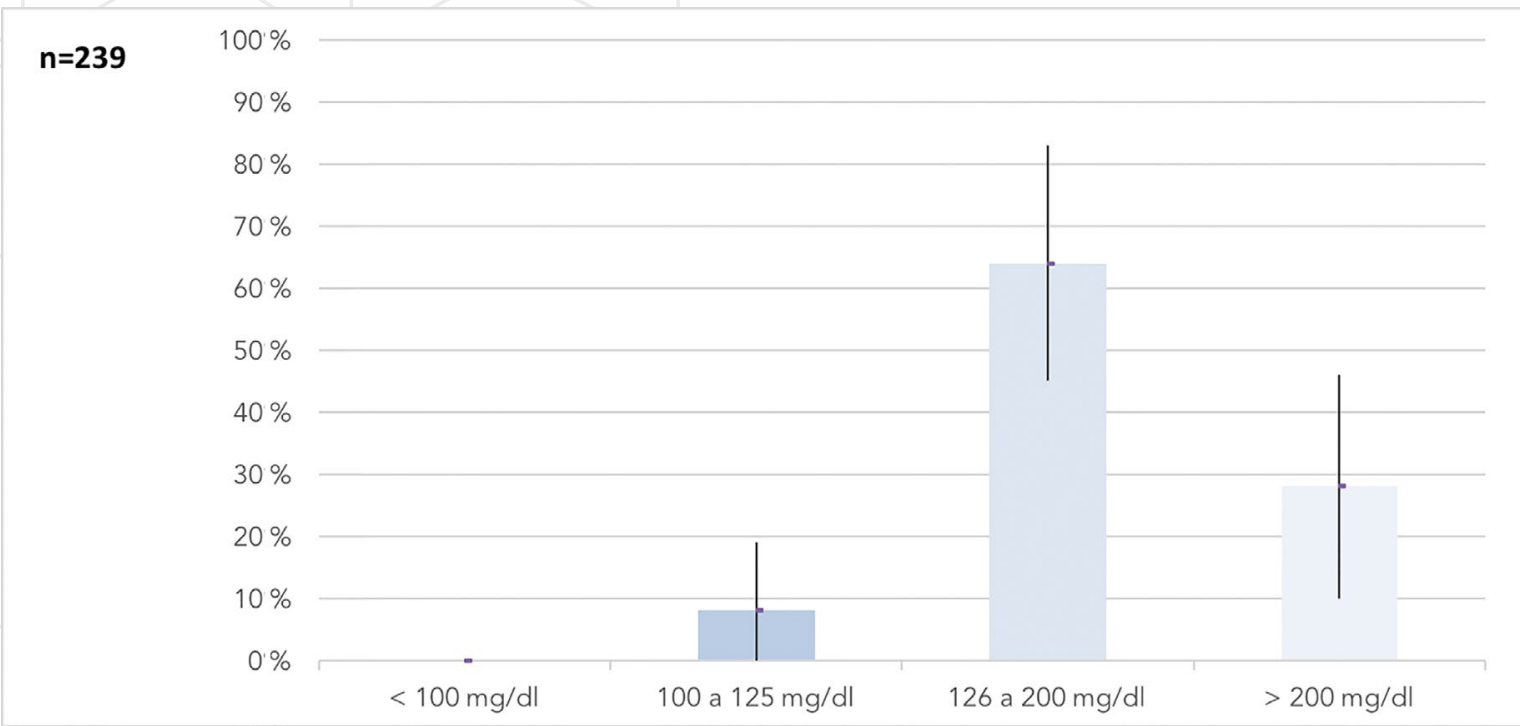

Fuente: informe final de tesis. 


\section{Discusión}

El presente informe corresponde a un estudio realizado a 239 entrevistados residentes de la aldea ya señalada. Se describen las variables sociodemográfica y clínicas de los factores de riesgo para diabetes mellitus de todos(as) los entrevistados(as). Se describe la puntuación de riesgo de padecer diabetes mellitus en 10 años por medio de la escala Findrisc. En conjunto con la caracterización del comportamiento de glicemia capilar, en la población con una puntuación igual o mayor a 15.

La caracterización

sociodemográfica de la

población es de suma

importancia, ya que con esta se puede obtener un registro de información que permite reconocer el entorno social y económico de una persona, lugar o este caso de una comunidad, para así conocer los aspectos de su situación actual. Con el propósito de poner en contexto a quienes deseen utilizar o replicar el presente estudio.

Respecto a la caracterización sociodemográfica, en la población estudiada el género femenino, fue el predominante (87\%), se obtuvo una razón de hombres, mujeres de 1:7 (tabla 1). Cabe resaltar que las entrevistas fueron realizadas en las viviendas de los participantes dentro del horario de 8:00 a 15:00 horas, con lo cual predominó la población femenina, debido a los roles culturales de la localidad.

En relación con la prevalencia de etnicidad la población maya supera de manera significativa a la población ladina, se tuvo una razón existente de ladino, maya de 1:6 (tabla 1). Es importante recordar que la aparición temprana de la enfermedad ocurre en la población mestiza; la población no indígena, además, posee mayor prevalencia de resistencia a la insulina en comparación con la pertenencia indígena.

Respecto a la caracterización de variables clínicas, este estudio demuestra prevalencia de factores de riesgo para diabetes mellitus, a través de la escala Findrisc que consta de 8 preguntas. En la mayoría de los estudios la edad es uno de los principales factores asociados al desarrollo de la diabetes, es más alta la prevalencia de esta enfermedad en personas mayores de 45 años (7). Sin embargo, la población estudiada presentaba una pirámide poblacional de base ancha, que limitó los resultados a una población predominantemente joven. Como resultado se obtuvo que, el $80 \%$ de la población no presenta riesgo en relación con la edad (tabla 2).

Respecto a las características clínicas encontradas, tan solo el $33 \%$ de la población se encontró dentro de un peso adecuado para la estatura, lo que categorizó a la población estudiada, con $36 \%$ en sobrepeso, $24 \%$ en obesidad grado I y $6 \%$ en obesidad grado II (figura 1). La Federación Internacional de Diabetes (FID) estima que, para el 2020, Guatemala se encontrará como uno de los seis países con mayor prevalencia de obesidad del mundo. Está sobreentendido que la obesidad es un factor de riesgo para el desarrollo de diabetes. Según la Organización Mundial de la Salud (OMS), el $74 \%$ de los casos con diabetes a nivel mundial se habrían evitado con un índice de masa corporal menor a $25 \mathrm{~kg} / \mathrm{m}^{2}$. Cabe destacar en los resultados que se muestran en la tabla 3, que aunque el consumo de frutas, verduras y hortalizas no fueron estadísticamente significativos en relación con el estado nutricional en la población estudiada, si se evidenció que la población que realizaba actividad física, independientemente de duración y la intensidad de la actividad, era 2.1 veces menos propensa, a presentar un IMC $>30 \mathrm{~kg} / \mathrm{m}^{2}$ $(p=0.05)$, aún más interesante se evidenció que las personas que consumen aguas edulcoradas, eran 2.2 veces más propensas a presentar un IMC superior $>25$ $\mathrm{kg} / \mathrm{m}^{2}(\mathrm{p}=0.0059)$. Aunque el IMC es un indicador muy preciso, cuenta con la desventaja que población robusta o fornida pueda ser erróneamente clasificada, por lo que se recomienda complementar la información con otros indicadores como el perímetro de cintura, que es un predictor más confiable de adiposidad central y que además está relacionada con resistencia a la insulina. 
Respecto a la obesidad abdominal central que se evaluó con el perímetro abdominal. El $66 \%$ del género masculino presentó un perímetro abdominal normal menor a $94 \mathrm{~cm}$, en contraste al género femenino, el $88.5 \%$ presentó un perímetro mayor a 80 y más del $50 \%$ de la población femenina presentó un aumento sustancial de riesgo con un perímetro abdominal mayor a $88 \mathrm{~cm}$ (figura 2). En la tabla 4 se evidencia que conductas poco saludables como el sedentarismo y el consumo de aguas edulcoradas, se relacionan con un perímetro abdominal mayor a $80 \mathrm{~cm}$, en la población femenina, incluso las mujeres que no realizan actividad física moderada por al menos 150 minutos son 3.7 veces más propensas a tener un perímetro abdominal inadecuado $(p=0.0001)$.

En relación con los antecedentes de hiperglicemia, $6 \%$ de población se le detectó al menos en una ocasión un episodio de hiperglicemia. La figura 3 lo muestra como un dato alentador para la comunidad, expresándose en menor riesgo de desarrollar diabetes mellitus, sin embargo, la población es $100 \%$ rural y depende en su totalidad del primer nivel del sistema de salud. Es decir, que el sistema sanitario de la localidad tiene limitados recursos, como escasas mediciones previas de glicemia capilar. Ambos factores pueden sesgar el resultado del estudio.
Con respecto al antecedente familiar diabetes mellitus, se encontró que el $28 \%$ poseen un familiar diagnosticado (figura 8). De acuerdo al grado de consanguinidad el $12 \%$ posee al menos un familiar de primera grado y $15 \%$ tiene familiares de segundo grado. Es bien conocido que la diabetes mellitus tipo 2 (DM2) es una enfermedad de predisposición genética, y la presencia de factores ambientales permiten su desarrollo y evolución. Se ha demostrado que la presencia de un familiar de primer grado (padres, hermanos e hijos(as)) es un factor significativo para el desarrollo de la misma. Por lo que se puede decir que, más de $1 / 4$ de la población estudiada es 10 veces más propensa al desarrollo de DM independientemente de los estilos de vida.

Dado que la población femenina fue del 87 \% con relación al total de la población estudiada, se decidió caracterizarla de forma independiente. Se puede observar en la tabla 5 que es predominantemente joven, es decir, que el $71 \%$ es menor de 45 años. Respecto a estilos de vida, más de $3 / 4$ de la población es sedentaria y no realiza ningún tipo de actividad física fuera de sus tareas domiciliarias, solo el $21 \%$ consume diariamente frutas, verduras y hortalizas, además, el 36 \% refirió consumir diariamente aguas edulcoradas, lo que es congruente con los resultados obtenidos en relación al IMC y el perímetro abdominal, que demostró que el $58 \%$ de las mujeres se encontraba en sobrepeso u obesidad, con resultados aún más preocupante en relación con el perímetro abdominal, siendo un $77 \%$, las que presentaron un perímetro mayor a $80 \mathrm{~cm}$. 
En la figura 5 se puede observar la prevalencia de los factores de riesgo evaluados en la escala de Findrisc, se encontró que el aumento en el perímetro abdominal, la escasa actividad física y el pobre consumo de frutas, verduras y hortalizas, fue del $82 \%$ en la población. Cabe destacar que los 3 factores son modificables y pueden ser corregidos por las y los entrevistados con ayuda del personal médico.

Con base en la escala de Findrisc, se puede observar que en la figura 6, que la categoría más frecuente obtenida fue la de riesgo ligeramente elevado, lo que significa que la posibilidad de desarrollar diabetes mellitus es de 1 en 25 , y que la población con alto riesgo que no fue mayor del $16 \%$ del total de la muestra, resultado que es parcialmente alentador, debido a que la población estudiada es prevalentemente joven.

A todo paciente que obtenían un resultado de 15 puntos o mayor en la escala Findrisc, se le realizó la prueba de glucosa capilar en ayunas, lo que evidenció tabla 7, que ninguna presentó una glicemia por debajo de 100 mg/ $\mathrm{dl}$, es decir, que todos(as) las y los pacientes de alto riesgo se encuentran con un anormal comportamiento de su glicemia y deberían de ser referidos(as) a un centro sanitario, que tenga la capacidad de hacer pruebas diagnósticas para diabetes mellitus. Este resultado guarda relación con el punto de cohorte (15 puntos) establecido en las guías europeas para hacer pruebas invasivas (glucosa sérica o HgA1C). Además, el $40 \%$ de la población de alto riesgo presentó una glicemia capilar en ayuno $>180 \mathrm{mg} / \mathrm{dl}$, lo que quiere decir que la concentración de glucosa ya se encuentra a niveles tóxicos, ello sugiere que esta población ya está cursando con daños metabólicos importantes.

En relación con el consumo de aguas edulcoradas, resultado que se muestra en la figura 8 , el $81 \%$ de la población consume al menos una vez por semana aguas edulcoradas, de la cual el $44 \%$ refirió consumirla diariamente, mientras que tan solo un $19 \%$ dijo no ingerir semanalmente ese producto. De hecho, se pudo identificar que el consumo de las mismas, presentó una asociación estadísticamente significativa ( $p$ valor $<0.05$ ) con la presencia de sobrepeso $(p=0.0059)$ y de un perímetro abdominal con aumento sustancial de riesgo $(p=0.032)$. Además, el OR indicó que la población que consume aguas edulcoradas en relación con los que no consumen son 2.2 veces más propensos al presentar un IMC > $5 \mathrm{~kg} / \mathrm{m}^{2}$, así como 1.9 veces más tendencia a presentar un perímetro abdominal mayor a $88 \mathrm{~cm}$ en mujeres y $102 \mathrm{~cm}$ en hombres.

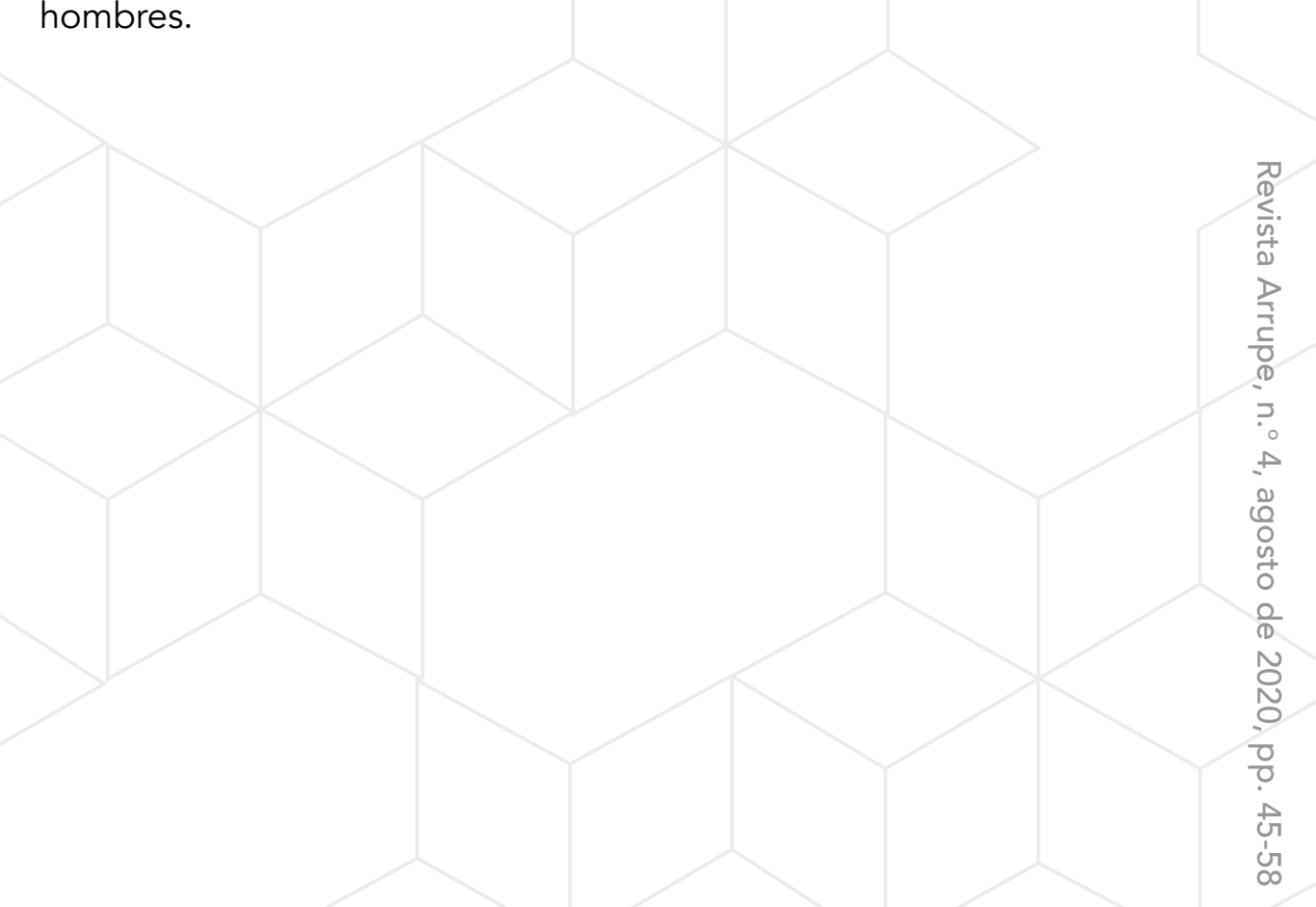




\section{Referencias}

1. International Diabetes Federation. Diabetes Atlas, Bruselas, Bélgica: International Diabetes Federation. 2013. Disponible en: http://www.idf.org/ diabetesatlas

2. Guía de la Asociación Latinoamericana de Diabetes sobre el diagnóstico y tratamiento de la diabetes mellitus tipo 2 con medicina basada en evidencia. Revista de la Asociación Latinoamericana de Diabetes. Colombia: ALAD, 2013.

3. Roglic G. WHO Global report on diabetes: A summary. International Journal of Noncommunicable Diseases. 2016;1(1):3. Disponible en: http://apps.who.int/ iris/bitstream/10665/204871/1/9789241565257_eng.pdf

4. Chamberlain J, Rhinehart A, Shaefer C, Neuman, A. Diagnosis and Management of Diabetes: ADA Standards of Medical Care in Diabetes. Annals of Internal Medicine, 2016;164(8):542-552.

5. Soriguer F, Valdés S, Tapia M. Med Validación del Findrisc (FINnish Diabetes Risk SCore) para la predicción del riesgo de diabetes tipo 2 en una población del sur de España. Estudio Pizarra Clin (Barc). 2012;138(9):371-376. Disponible en: http://www.elsevier.es

6. Goldman L. - Diabetes Mellitus Tipo 1. Enfermedades Endocrinas. En Tratado de Medicina Interna. 24. ${ }^{a}$ ed. (p. 1479). España: Elsevier; 2013. vol. 2, sección XVIII.

7. Ruano M. Prevalencia de diabetes y prediabetes y su relación con la puntuación de riesgo Findrisc en los trabajadores de una empresa de fabricación de resortes de suspensión para transportes pesados y livianos. Tesis doctoral. Guatemala: Universidad Europea del Atlántico; 2017. 